\title{
Estudio de la ansiedad en el paciente prequirúrgico en cirugía del pie
}

\author{
Anxiety study in the presurgical patient in foot surgery \\ Daniel Navarro-Gastón \\ Departamento de Podología, Universidad de Sevilla. Sevilla, España
}

Palabras clave:

Ansiedad preoperatoria, cirugía del pie, cirugía podológica, desinformación

\section{Resumen}

Introducción: El estado emocional del paciente antes de una cirugía del pie no está incluido en la valoración prequirúrgica rutinaria en Podología. La ansiedad preoperatoria ha sido estudiada en diferentes disciplinas médicas, pero se desconoce en procedimientos quirúrgicos del pie. El objetivo principal fue determinar la prevalencia de ansiedad preoperatoria en cirugía del pie, así como evaluar la demanda de información quirúrgica de los pacientes.

Pacientes y métodos: Se empleó la escala validada de ansiedad e información de Ámsterdam (APAIS) para evaluar la ansiedad preoperatoria y la necesidad de información en 93 pacientes sometidos a diferentes cirugías del antepié (osteoarticular, ungueal y partes blandas). También se utilizó un cuestionario para la recogida de otras variables de interés antes de la cirugía como la edad, sexo y nivel educativo.

Resultados: La ansiedad preoperatoria se presentó en el $23.7 \%$ de los pacientes y el $49.5 \%$ demandó más información. Además, existió relación entre ambas variables $(p<0.05)$. La ansiedad fue mayor en las mujeres $(p=0.023)$. La ansiedad y la demanda de información no se relacionaron con el resto de las variables.

Conclusión: En este estudio existió ansiedad y desinformación en cirugía podológica. Por tanto, existe la necesidad de instaurar la valoración psicológica prequirúrgica e informar al paciente adecuadamente.

Keywords:

Preoperative anxiety, foot surgery, podiatric surgery, misinformation.

\section{Abstract}

Introduction: The emotional state of the patient before a foot surgery is not included in the routine presurgical assessment in Podiatry. The assessment of preoperative anxiety has been studied in different medical disciplines, but it is unknown in surgical procedures of the foot. The main objective was to determine the prevalence of preoperative anxiety in foot surgery, as well as to assess the demand for surgical information from patients.

Patients and methods: The validated Amsterdam anxiety and information scale (APAIS) was used to assess preoperative anxiety and the need for information in 93 patients undergoing different forefoot surgery (osteoarticular, nail and soft tissue). A questionnaire was also used to collect other variables before surgery such as age, sex and educational level.

Results: Preoperative anxiety occurred in $23.7 \%$ of the patients and $49.5 \%$ demanded more information. In addition, there was a relationship between both variables $(p<0.05)$. Anxiety was higher in women $(p=0.023)$. Anxiety and demand for information were not related to the rest of the variables.

Conclusion: In this study there was high anxiety and misinformation in podiatric surgery. Therefore, there is a need to establish the pre-surgical psychological assessment and inform the patient properly. 


\section{INTRODUCCIÓN}

En la actualidad, cada vez es mayor el número de procedimientos quirúrgicos en cirugía ambulatoria al año por sus numerosas ventajas, entre ellas la no hospitalización, el menor coste y una mejor recuperación posoperatoria. A pesar de ello, cualquier cirugía es un acontecimiento estresante que frecuentemente puede desencadenar en un proceso de ansiedad. La ansiedad es una respuesta esperada y uno de los problemas más importantes para los pacientes, porque causa problemas emocionales y físicos durante el proceso quirúrgico'.

En Podología, cada vez son más los profesionales que realizan intervenciones quirúrgicas, pero la valoración del estado psicológico del paciente antes y durante la intervención suele ser subestimada por el profesional. La etiología y el nivel de ansiedad es diversa. Los pacientes que refieren tener ansiedad antes de la cirugía, independientemente del resultado de la escala, suele ser causada por la anticipación del dolor, el miedo a los procedimientos quirúrgicos y/o anestésicos, la posibilidad de cambios en la imagen corporal e incluso la muerte 2 .

En la última década se ha demostrado que la ansiedad influye negativamente en los procedimientos quirúrgicos, siendo un factor contribuyente en los resultados quirúrgicos y en la evolución adecuada del posoperatorio ${ }^{1,3}$. Por ello, es fundamental valorar y detectar la ansiedad preoperatoria para ayudar al paciente y evitar que afecte en cualquier momento del proceso quirúrgico. Determinar el nivel de ansiedad en cirugía podológica nos permitiría saber si existe la necesidad de incluir la evaluación del estado emocional del paciente en la valoración preoperatoria.

En la literatura no existen estudios específicos desde nuestro conocimiento que aborden el objetivo principal de este estudio, determinar la prevalencia de ansiedad preoperatoria en cirugía del pie y el nivel de demanda de información del paciente.

\section{PACIENTES Y MÉTODOS}

Tipo de diseño

Se realizó un estudio observacional durante el periodo comprendido entre enero y diciembre de 2017, con un muestreo no probabilístico por conveniencia y consecutivo de los pacientes programados para cualquier intervención quirúrgica del pie en el Área Clínica de Podología de la Universidad de Sevilla y que firmaron el consentimiento. El estudio fue aprobado por el Comité de ética de los Hospitales Universitarios Virgen de la Macarena y Virgen del Rocío de Sevilla (España), con código interno 0859-N-18.

\section{Participantes}

En este estudio se ha obtenido un total de 93 pacientes sometidos a procedimientos de cirugía ungueal, partes blandas y osteoarticular. Los criterios de inclusión fueron: ser mayor de 18 años, ASA I y II (American Society of Anesthesiologists), con capacidad autónoma para rellenar las encuestas y participar en el estudio. Se excluyeron del estudio los pacientes con trastornos mentales y/o emocionales, con deterioro cognitivo, con incapacidad para comprender y responder a los cuestionarios y aquellas personas medicadas con ansiolíticos antes de la cirugía.

\section{Recogida de datos}

La recogida de datos comenzaba en la sala de espera para captar el momento de mayor ansiedad, como señalan algunos autores ${ }^{1}$. Los participantes completaban un cuestionario específico que contenía los datos sociodemográficos y la Escala Preoperatoria de Ansiedad e Información de Ámsterdam (APAIS), la cual permite evaluar la ansiedad preoperatoria del paciente programado para cirugía, así como la demanda de más información quirúrgica ${ }^{4,5}$.

La escala APAIS consta de seis afirmaciones que se dividen en dos subapartados, uno mide la ansiedad del paciente respecto a la anestesia y la cirugía, y el segundo mide el deseo de más información sobre la anestesia y la cirugía. Las respuestas a las afirmaciones se evaluaron con una escala Likert (1: En absoluto, a 5: Extremadamente). Los puntos de corte establecidos por los autores de la versión original para determinar ansiedad clínica en un paciente son $\geq 11^{4}$. Además, recomiendan dar más información sobre lo que desean estar informados a los pacientes con una puntuación $\geq$ a 5 .

La explicación del estudio a los participantes y recogida de datos fue realizada por un único investigador.

\section{Análisis estadístico}

El análisis de los datos se realizó mediante el programa estadístico IBM ${ }^{\circledast}$ SPSS Statistics versión 25 para Windows 10 (IBM Corp, Armonk, New York).

El coeficiente $\alpha$ - Cronbach se calculó para evaluar la consistencia interna de las dos subescalas. Se consideró aceptable cuando el $\alpha$ de Cronbach era $>0.70$.

Se realizó un análisis descriptivo de las variables cuando fueron de interés para el estudio. Las pruebas de normalidad mostraron que los datos no siguieron una distribución normal. Por tanto, se empleó la prueba de la U de Mann-Whitney para las comparaciones entre 2 grupos y la prueba de Kruskall-Wallis para las comparaciones entre 3 o más grupos. Las correlaciones se estudiaron mediante la prueba de la Rho de Spearman y Tau b de Kendall. Se consideró significativo cualquier valor de $p$ menos o igual que 0.05 .

\section{RESULTADOS}

Un total de 93 pacientes fueron analizados en el estudio, 78 mujeres y 15 hombres. La edad de los pacientes osciló entre 19 y 91 años con una media de 54.4 años, siendo simi- 


\begin{tabular}{|c|c|c|}
\hline & & $\begin{array}{c}\text { Características } \\
\text { de la muestra } \\
n(\%)\end{array}$ \\
\hline \multirow{2}{*}{ Sexo } & Hombre & $15(83.9 \%)$ \\
\hline & Mujer & $78(16.1 \%)$ \\
\hline \multirow{3}{*}{ Edad } & $18-<41$ & $17(18.3 \%)$ \\
\hline & $41-<65$ & $47(50.5 \%)$ \\
\hline & $\geq 65$ años & $29(31.2 \%)$ \\
\hline \multirow{6}{*}{ Nivel de estudios } & Ninguno & $11(11.8 \%)$ \\
\hline & Primaria & $18(19.3 \%)$ \\
\hline & Secundaria & $16(17.2 \%)$ \\
\hline & Bachillerato & $5(5.4 \%)$ \\
\hline & C. Formativo & $13(14.0 \%)$ \\
\hline & Universidad & $30(32.3 \%)$ \\
\hline \multirow{3}{*}{ Tipo de cirugía } & Ungueal & $61(65.6 \%)$ \\
\hline & Osteoarticular & $20(21.5 \%)$ \\
\hline & Partes blandas & $12(12.9 \%)$ \\
\hline
\end{tabular}

lares entre hombres y mujeres. Otras características demográficas están recogidas en la Tabla I.

La fiabilidad interna de APAIS fue buena. Se obtuvo para los ítems que miden la ansiedad un alfa de Cronbach de 0.87, y 0.86 para los ítems que miden la necesidad de más información.

En la Tabla II se recoge la distribución de los pacientes catalogados con ansiedad según las variables analizadas. El $23.7 \%(n=22)$ presentó algún grado de ansiedad, siendo muy elevado en 8 personas con una puntuación $\geq a 16$.

Se encontró diferencia significativa entre la ansiedad y el sexo ( $p=0.023)$. Es decir, las mujeres presentaron más ansiedad que los hombres. En cambio, no se encontró entre la ansiedad y los diferentes rangos de edad, los diferentes niveles educativos o el tipo de cirugía.

En la Figura 1 se muestran las diferentes causas de los 44 pacientes que referían encontrarse nerviosos antes de la cirugía independientemente del resultado de APAIS.

Se encontró diferencia significativa $(p<0.001)$ y correlación con la ansiedad (Tau b de Kendall $=0.386$ ), es decir, los pacientes que refirieron estar nerviosos presentaron mayor nivel de ansiedad, con una media de 10.41 (SD \pm 4.8 ) frente a 6.5 (SD \pm 3.1 ) de los sujetos que no refirieron nerviosismo.

En la Tabla III se muestran los pacientes según el deseo de más información. El 49.5 \% de la muestra demandó más información, en concreto 6 hombres (6.5\%) y 40 mujeres (43\%).

No se hallaron diferencias significativas entre la demanda de información y las variables sexo, edad, nivel de estudios

\begin{tabular}{|c|c|c|}
\hline & Muestra total $(n=93)$ & $\begin{array}{c}\text { Pacientes con } \\
\text { ansiedad } \\
\mathrm{n}(* \%)\end{array}$ \\
\hline \multirow{3}{*}{ Edad } & $18-<41$ años * $(n=17)$ & $4(23.5 \%)$ \\
\hline & $41-<65$ años * $(n=47)$ & $11(23.4 \%)$ \\
\hline & $\geq 65$ años * $(n=29)$ & $7(24 \%)$ \\
\hline \multirow{2}{*}{ Sexo } & Hombre * $(n=15)$ & $1(6.6 \%)$ \\
\hline & Mujer $*(n=78)$ & $21(26.9 \%)$ \\
\hline \multirow{6}{*}{$\begin{array}{l}\text { Nivel de } \\
\text { estudios }\end{array}$} & Ninguno * $(n=11)$ & $3(27.7 \%)$ \\
\hline & Primaria * $(n=18)$ & $6(33.3 \%)$ \\
\hline & Secundaria * $(n=16)$ & $1(6.3 \%)$ \\
\hline & Bachillerato * $(n=5)$ & $3(60 \%)$ \\
\hline & C. Formativo * $(n=13)$ & $3(23.1 \%)$ \\
\hline & E. Superiores * $(n=30)$ & $6(20 \%)$ \\
\hline \multirow{3}{*}{ Tipo de cirugía } & Ungueal * $(n=61)$ & $13(20 \%)$ \\
\hline & Osteoarticular * $(n=20)$ & $6(30 \%)$ \\
\hline & Partes blandas * $(n=12)$ & $3(25 \%)$ \\
\hline
\end{tabular}

*Porcentaje respecto al número de sujetos que componen cada subgrupo de la variable analizada.

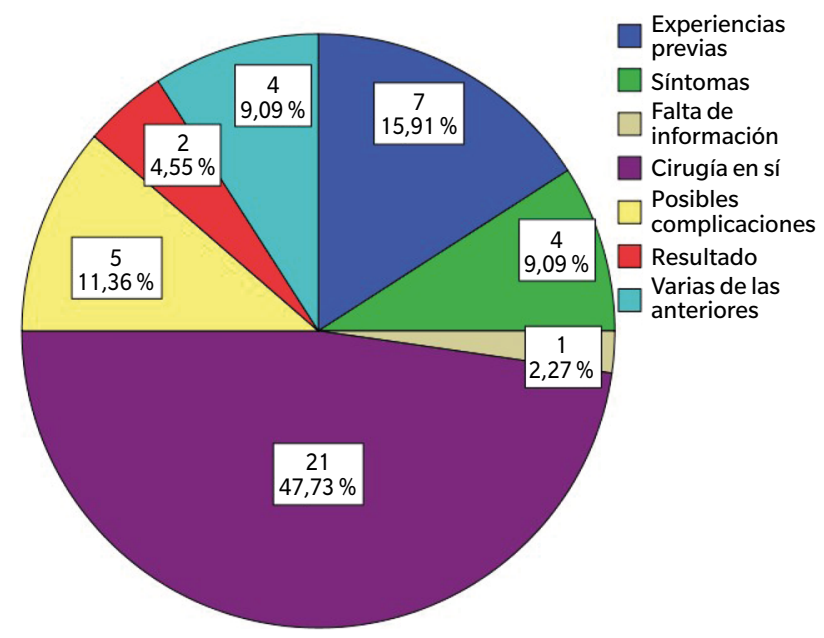

Figura 1. Causas del nerviosismo referido por los pacientes.

o tipo de cirugía. En cambio, la necesidad de más información se correlacionó con la ansiedad ( $r=0.258, p=0.013)$. Es decir, los pacientes que tuvieron más ansiedad demandaron más información sobre el procedimiento anestésico-quirúrgico o viceversa. La diferencia se encontró entre los sujetos que no demandaban nada de información y los que demandaban alta información $(p=0.018)$. En la Tabla IV se muestra la distribución de los pacientes según la ansiedad y la necesidad de más información. 
Tabla III. Distribución de los pacientes según la demanda de información y las diferentes variables.

\begin{tabular}{|c|c|c|c|c|c|}
\hline & & \multicolumn{4}{|c|}{ Necesidad de Información } \\
\hline & & $\begin{array}{l}\text { No precisa } \\
(\mathrm{n}=47)\end{array}$ & $\begin{array}{l}\text { Moderada } \\
(\mathrm{n}=30)\end{array}$ & $\begin{array}{l}\text { Elevada } \\
(n=16)\end{array}$ & $\begin{array}{c}\text { Total moderado- } \\
\text { elevada }\end{array}$ \\
\hline & Muestra total $(n=93)$ & $n(* \%)$ & $\mathrm{n}$ & $\mathrm{n}$ & $n(* \%)$ \\
\hline \multirow{3}{*}{ Edad } & $18-<41$ años * $(n=17)$ & $9(52.9)$ & 4 & 4 & $8(47.1)$ \\
\hline & $41-<65$ años * $(n=47)$ & $19(60.4)$ & 19 & 9 & $28(59.6)$ \\
\hline & $\geq 65$ años * $(n=29)$ & $19(65.5)$ & 7 & 3 & $10(34.5)$ \\
\hline \multirow{2}{*}{ Sexo } & Masculino * $(n=15)$ & $9(45.5)$ & 4 & 2 & $6(54.5)$ \\
\hline & Femenino $*(n=78)$ & $38(38.9)$ & 9 & 2 & $11(61.1)$ \\
\hline \multirow{6}{*}{$\begin{array}{l}\text { Nivel de } \\
\text { estudios }\end{array}$} & Ninguno * $(n=11)$ & $5(75)$ & 3 & 1 & $4(25)$ \\
\hline & Primaria * $(n=18)$ & $7(40)$ & 1 & 2 & $3(60)$ \\
\hline & Secundaria * $(n=16)$ & $12(38.5)$ & 6 & 2 & $8(61.5)$ \\
\hline & Bachillerato * $(n=5)$ & $2(53.3)$ & 7 & 7 & $14(46.7)$ \\
\hline & C. Formativo * $(n=13)$ & $5(52.9)$ & 4 & 4 & $8(47.1)$ \\
\hline & E. Superiores * $(n=30)$ & $16(40.4)$ & 19 & 9 & $28(59.6)$ \\
\hline \multirow{3}{*}{$\begin{array}{l}\text { Tipo de } \\
\text { cirugía }\end{array}$} & Ungueal * $(n=61)$ & $29(47.5)$ & 22 & 10 & $32(52.5)$ \\
\hline & Osteoarticular * $(n=20)$ & $12(60)$ & 6 & 2 & $8(40.0)$ \\
\hline & Partes blandas * $(n=12)$ & $6(50)$ & 2 & 4 & $6(50.0)$ \\
\hline
\end{tabular}

*Porcentaje respecto al número de sujetos que componen cada subgrupo de la variable analizada.

\begin{tabular}{llccccc}
\multicolumn{1}{c}{ Tabla IV. Distribución de la muestra según la ansiedad $\mathbf{y}$ demanda de información. } & & \\
& $\begin{array}{c}\text { No precisa } \\
\mathrm{n}\end{array}$ & $\begin{array}{c}\text { Total } \\
(\%)\end{array}$ & $\begin{array}{c}\text { Moderada } \\
\mathrm{n}\end{array}$ & $\begin{array}{c}\text { Alta } \\
\mathrm{n}\end{array}$ & $\begin{array}{c}\text { Total * } \\
\mathrm{n}(\%)\end{array}$ \\
\hline \multirow{2}{*}{ Ansiedad } & $\mathrm{No}^{*}(\mathrm{n}=71)$ & 39 & $54.9 \%$ & 23 & 9 & $32(45.1 \%)$ \\
\cline { 2 - 7 } & $\mathrm{Si}^{*}(\mathrm{n}=22)$ & 8 & $36.4 \%$ & 7 & 7 & $14(63.6 \%)$ \\
\hline
\end{tabular}

*Porcentaje respecto al número de sujetos que componen cada subgrupo de la variable analizada.

\section{DISCUSIÓN}

El objetivo principal de este trabajo fue evaluar la ansiedad preoperatoria en diferentes cirugías del pie. Para ello, se utilizó el sistema de puntuación APAIS validado al español y diseñado para la cirugía electiva. Los resultados de fiabilidad de APAIS fueron aceptables y similares a lo obtenido en la versión original ${ }^{4}$ y en la versión española ${ }^{5}$.

El principal hallazgo del presente estudio muestra que casi uno de cada cuatro pacientes $(23.7 \%)$ presentó ansiedad prequirúrgica, siendo en el $36.4 \%$ de ellos elevado $(\geq 16)$. Esto es comparable a estudios previos que emplearon la escala APAIS como el realizado por Ivette y cols. ${ }^{6}$, quienes obtuvieron el que $25 \%$ de 100 pacientes con ansiedad sometidos a diversos tipos de cirugía y anestesia, con predominio de la colecistectomía laparoscópica, o Anguita y cols. ${ }^{7}$, quienes hallaron el $32 \%$ de 200 pacientes con ansiedad, siendo intervenidos con cirugía menor en 145 pacientes.

Otros estudios reportaron entre el 38 y el $94 \%$ en diferentes procedimientos quirúrgicos ${ }^{3,8-10}$. Estos datos tan ele- vados podrían ser explicados por la heterogeneidad de los estudios respecto a la muestra, herramientas y metodología empleada. La complejidad quirúrgica también es recogida como factor de riesgo de ansiedad preoperatoria ${ }^{6}$.

A diferencia con nuestro trabajo, Mandy y cols. ${ }^{11}$ estudiaron la evolución de los diferentes estados anímicos en 85 sujetos sometidos a diferentes cirugías del pie desde el periodo preoperatorio hasta 8 semanas después con diferentes escalas. Obtuvieron un estado de ansiedad moderado-alto del $54 \%$.

Respecto al sexo y la ansiedad, se encontró diferencia significativa $(p=0.023)$. Las mujeres presentaron mayor ansiedad que los hombres. Esto es reportado por varios estudios ${ }^{4,9,12}$. Entre las posibles razones podría estar que las mujeres expresan más libremente sus preocupaciones y temores.

Los resultados actuales muestran que la edad influye en la ansiedad prequirúrgica, siendo mayor en pacientes jóvenes ${ }^{8}$. Entre las razones, podría ser debido a que los jóvenes tienen una aceptación de la realidad más difícil. En cambio, 
al igual que en nuestros resultados, no se halló este hecho en otro trabajo ${ }^{9}$. Tampoco se encontró asociación entre el nivel de ansiedad y el nivel educativo ${ }^{13}$.

El principal motivo causante de ansiedad referido por los pacientes fue la propia intervención, con el $47.73 \%$. En la literatura, se recoge entre los principales motivos junto al éxito quirúrgico, síntomas, las agujas y anestesia general ${ }^{6,9,13}$. Otro hallazgo interesante fue que el $49.5 \%$ de la muestra demandó más información. Al igual que otros autores ${ }^{13-15}$, nuestros resultados muestran con significación estadística ( $p=0.018)$ que los pacientes que demandaron más información presentaron mayor ansiedad frente a los que no demandaban información.

La evidencia demuestra que las visitas preoperatorias y la disponibilidad de una persona para informar al paciente son efectivas para disminuir la ansiedad $d^{13,16}$. Pereira y cols. ${ }^{17}$ indicaron con significación estadística la eficacia de la entrevista o consulta preoperatoria para reducir la ansiedad prequirúrgica. Además, al igual que con otros autores ${ }^{18}$, a pesar de no encontrar diferencias significativas, obtuvimos que los pacientes más jóvenes demandaron más información que los adultos mayores de 65 años. Tampoco existió diferencias entre hombres y mujeres. Con respecto al nivel de estudios, algunos autores ${ }^{19}$ hallaron que los pacientes con niveles de estudios más altos demandaban más información, en nuestro trabajo no fue así. Esto podría ser porque pueden expresarse mejor y tienen mayor conciencia sobre la anestesia y la cirugía.

Los hallazgos de este estudio son novedosos porque se trata de un tipo de cirugía poco estudiada anteriormente, el cual nos permite conocer que existe ansiedad en procedimientos quirúrgicos menores del pie. Esto podría tenerse en cuenta para registrar sistemáticamente el nivel de ansiedad preoperatoria y tratarla. También, se podría aportar más información a los pacientes respetando su preferencia a ser informado en cuanto a nivel de información, cantidad y tiempo. La importancia de evaluar y detectar la ansiedad preoperatoria radica en que un alto grado de ansiedad puede aumentar la complejidad del procedimiento y afectar al desarrollo favorable de la cirugía y a la calidad asistencial. En el futuro, otros estudios podrían ir orientados a la influencia del tipo y forma que se proporciona la información al paciente respecto al nivel de ansiedad, o comprobar la eficacia de la medicación ansiolítica preoperatoria en comparación con otras alternativas para disminuir la ansiedad.

Este estudio tiene algunas limitaciones, como no disponer de mayor número de estudios similares que nos permita comparar mejor nuestros resultados, siendo difícil debido a la heterogeneidad de los trabajos respecto a la muestra, método y tipo de cirugía. Otra limitación fue la presencia de más mujeres que hombres, no pudiendo realizar comparaciones más equitativas. Finalmente, el obtener la muestra de un centro único nos impide generalizar resultados a otros centros sanitarios.
En conclusión, el presente estudio encontró que parte importante de la muestra presentó ansiedad preoperatoria en los diferentes procedimientos quirúrgicos menores del pie a los que fueron sometidos, siendo mayor en la mujer. Además, hubo una importante desinformación del paciente respecto al procedimiento quirúrgico, el cual se relacionó con aumento del nivel de ansiedad prequirúrgica. Por lo tanto, existe la necesidad de valorar y detectar con herramientas adecuadas la ansiedad, para tratarla y mejorar el proceso de atención al paciente.

\section{CONFLICTOS DE INTERESES}

No existen.

\section{FINANCIACIÓN}

No recibida.

\section{BIBLIOGRAFÍA}

1. Wilson CJ, Mitchelson AJ, Tzeng TH, El-Othmani MM, Saleh J, Vas$\operatorname{dev} \mathrm{S}$, et al. Caring for the surgically anxious patient: a review of the interventions and a guide to optimizing surgical outcomes. Am J Surg. 2016;212(1):151-9. DOI: 10.1016/j.amjsurg.2015.03.023.

2. Bailey L. Strategies for decreasing patient anxiety in the perioperative setting. AORN J. 2010;92(4):445-60. DOI: 10.1016/j.aorn.2010.04.017.

3. Turksal E, Alper I, Sergin D, Yüksel EA, Ulukaya S. The Effects of Preoperative Anxiety on Anesthetic Recovery and Postoperative Pain in Donor Nephrectomy. Transplantation. 2017;101:S116-7. DOI: 10.1097/01. tp.0000525160.85577.be.

4. Moerman N, Oosting H. The Amsterdam Scale (APAIS). Anesth Analg. 1996;82(3):445-51. DOI: 10.1097/00000539-199603000-00002.

5. Vergara M, Morales JM, Morales A, Canca JC, Rivas F, Reinaldo JA, et al. Validation of the Spanish version of the Amsterdam preoperative anxiety and information scale (APAIS). Health Qual Life Outcomes. 2017;15(1):1-10. DOI: 10.1186/s12955-017-0695-8.

6. Hernández Hernández IA, López BascopeJA, Guzmán SánchezJA. Nivel de ansiedad e información preoperatoria en pacientes programados para cirugía. Un estudio transversal descriptivo. Acta Médica Grup Ángeles. 2016;14(1):6-11.

7. Anguita MC, Talayerol M, Herrero S, Martín M, Pardo P, Gil A. Comportamientos de miedo y ansiedad prequirúrgicos e intensidad de dolor percibida tras una artroscopia de rodilla. Enferm Clin. 2016;26(4):22733. DOI: 10.1016/j.enfcli.2016.02.002.

8. Bradshaw P, Hariharan S, Chen D. Does preoperative psychological status of patients affect postoperative pain? A prospective study from the Caribbean. Br J Pain. 2016;10(2):108-15. DOI: 10.1177/2049463716635680.

9. Kuzminskaitè V, Kaklauskaitė J, Petkevičiūtè J. Incidence and features of preoperative anxiety in patients undergoing elective non-cardiac surgery. Acta Medica Litu. 2019;26(1):93-100. DOI: 10.6001/actamedica. v26i1.3961.

10. Hernández-Palazón J, Fuentes-García D, Falcón-Araña L, Roca-Calvo MJ, Burguillos-López S, Doménech-Asensi P, et al. Assessment of Preoperative Anxiety in Cardiac Surgery Patients Lacking a History of Anxiety: Contributing Factors and Postoperative Morbidity. J Cardiothorac Vasc Anesth. 2018;32(1):236-44. DOI: 10.1053/j.jvca.2017.04.044.

11. Mandy A, Feeney S. Changes in mood state after day case forefoot surgery. J Foot Ankle Surg. 2014;53(3):286-90. DOI: 10.1053/j. jfas.2014.01.011.

12. Garip H, Abalı O, Göker K, Göktürk Ü, Garip Y. Anxiety and extraction of third molars in Turkish patients. Br J Oral Maxillofac Surg. 2004;42(6):551-4. DOI: 10.1016/j.bjoms.2004.08.001. 
13. Jiwanmall M, Jiwanmall S, Williams A, Kamakshi S, Sugirtharaj L, Poornima K, et al. Preoperative anxiety in adult patients undergoing day care surgery: Prevalence and associated factors. Indian J Psychol Med. 2020;42(1):87-92. DOI: 10.4103/IJPSYM.IJPSYM_180_19.

14. Doñate M, Litago A, Monge Y, Martínez R. Aspectos de la información preoperatoria relacionada con la ansiedad del paciente programado para cirugía. Enferm Glob. 2015;14(37):170-80. DOI: 10.6018/eglobal.14.1.185281.

15. Wattier J, Barreau O, Devos P, Prevost S, Vallet B, Lebuffe G. Mesure de l'anxiété et du besoin d'informations préopératoire en six questions. Ann Fr Anesth Reanim. 2011;30(7-8):533-7. DOl: 10.1016/j. annfar.2011.03.010

16. Sadati L, Pazouki A, Mehdizadeh A, Shoar S, Tamannaie Z, Chaichian S. Effect of preoperative nursing visit on preoperative anxiety and postop- erative complications in candidates for laparoscopic cholecystectomy: a randomized clinical trial. Scand J Caring Sci. 2013;27(4):994-8. DOI: 10.1111/scs. 12022 .

17. Pereira L, Figueiredo-Braga M, Carvalho IP. Preoperative anxiety in ambulatory surgery: The impact of an empathic patient-centered approach on psychological and clinical outcomes. Patient Educ Couns. 2016;99(5):733-8. DOI: 10.1016/j.pec.2015.11.016

18. Won K, Gyeong B, Bong S, Hyeon L. Availability of preoperative anxiety scale as a predictive factor for hemodynamic changes during induction of anesthesia. Korean J Anesthesiol. 2010;58(4):328-33. DOI: 10.4097/ kjae.2010.58.4.328.

19. Celik F, Edipoglu IS. Evaluation of preoperative anxiety and fear of anesthesia using APAIS score. Eur J Med Res. 2018;23(1):41. DOI: 10.1186/ s40001-018-0339-4. 